\title{
EMPLEO CON PROFESIONALIZACIÓN EN EL SECTOR TURISMO
}

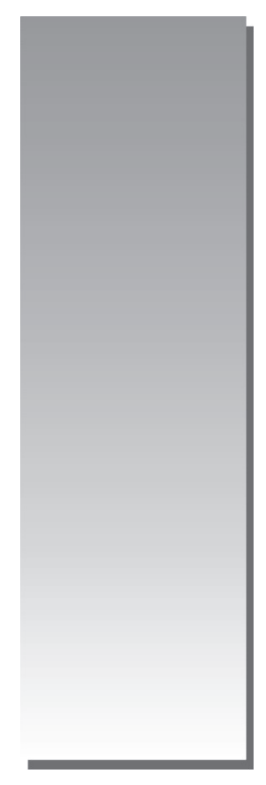

Mauro Granados Maguiño*

E-mail: granadosmauro@gmail.com

\begin{abstract}
RESUMEN
Parte del problema es que un empleo de baja calidad en el sector turístico implica proporcionalmente igual calidad en el servicio, además de una baja productividad global del sector y un riesgo inminente para su desarrollo en un contexto marcado por una creciente competencia. Si la masa laboral no está calificada adecuadamente, el nivel de la prestación será deficiente; por ello es necesario que los niveles de profesionalización sean altos y que los trabajadores hayan recibido formación y capacitación formal y académica en mayor proporción.
\end{abstract}

Palabras clave: Turismo, Educación, Empleo, Competitividad, Profesionalización, Experiencia, Especialización, Ventajas Competitivas.

\section{ABSTRACT}

Part of the problem is that a low quality employment in the tourist sector involves proportionally equal quality in the service. It is also a problem low global productivity of the sector and an imminent risk for its development in a context marked by an increasing competition. If the labor mass is not qualified adequately, the level of the service will be deficient; that is why it is necessary high levels of professionalization and workers have received formal and academic training in major proportion.

Key words: Tourism, Education, Employment, Competitiveness, Professionalization, Experience, Specialization, Competitive Advantages.

\footnotetext{
* Magister en Marketing Turístico y Hotelero (USMP). Licenciado en Turismo y Hotelería. Director de Escuela de Turismo de la Universidad Cristina de Bolivia, Director de la Escuela de Administración de Turismo (UNMSM), Decano del Colegio de Licenciados en Turismo del Perú.
} 


\section{INTRODUCCIÓN}

El turismo en el mundo, desde hace más de una década, viene presentando un crecimiento sostenido, más allá de los fenómenos coyunturales, constituyéndose en un gran generador de empleo y provisor de divisas para las economías de los países. El turismo concentra el $8.4 \%$ del empleo mundial, es decir 230 millones de empleos.

El turismo en el Perú presenta un incremento sostenido desde hace nueve años y, aún con dificultades externas, se estima que en el 2009 el sector creció en un 5\%; por su parte, el turismo interno también muestra un incremento sostenido. Podemos decir entonces que las divisas y la moneda nacional provenientes del turismo contribuyen al desarrollo nacional y apoyan el proceso de descentralización.

Tradicionalmente nuestro país ha basado su desarrollo en las ventajas comparativas en general, y en la actividad turística, en particular. Actualmente se deben desarrollar ventajas competitivas, para lo cual es vital contar con recursos humanos altamente calificados y competitivos. La masa trabajadora del sector turismo en el país alcanza los 400,000 empleos directos e indirectos; por tanto, es de suma importancia tener y mantener niveles de calidad en la prestación del servicio, considerando que en la actividad turística todo es servicio.

Hay que destacar que la masa laboral está conformada por trabajadores formados y forjados en el empleo mismo; es decir, personas que han aprendido por la experiencia, esto en mayor proporción en el interior del país, y que en algunos casos han recibido capacitación en asuntos específicos.

Por otro lado, en forma permanente y gradual, se van incorporando al mercado laboral profesionales egresados de instituciones educativas, universidades e institutos superiores tecnológicos que brindan formación en turismo, hotelería, gastronomía y ecoturismo. Existen además otras instituciones que ofertan capacitación de corta duración en diversos temas y aéreas del turismo. Por ello es importante conocer las condiciones del empleo en turismo así como el espectro de la formación en turismo, a nivel nacional.

Parte del problema es que un empleo de baja calidad en el sector turístico implica proporcionalmente igual calidad en el servicio, además de una baja productividad global del sector y un riesgo inminente para su desarrollo en un contexto marcado por una creciente competencia. Si la masa laboral no está calificada adecuadamente, el nivel de la prestación será deficiente; por ello es necesario que los niveles de profesionalización sean altos y que los trabajadores hayan recibido formación y capacitación formal y académica en mayor proporción.

Si se considera que el problema principal que acarrea la falta de profesionalización es el bajo nivel del servicio, hay que contar con una mayor proporción de empleados calificados en lugar de personal empírico, para marcar así la diferencia con empresas turísticas de otros países. El principal problema que tienen las diferentes empresas turísticas en el país es conseguir personal calificado y especializado dentro de los diferentes rubros turísticos, lo que no es fácil de lograr.

\section{LOS RECURSOS HUMANOS EN LA ACTIVIDAD TURÍSTICA}

La globalización de los mercados y la apertura de las economías demandan modelos de gestión flexibles. En ellos la inteligencia, la innovación y la creatividad, apoyadas por la tecnología y la productividad generadas son fundamentales para el establecimiento de las nuevas relaciones. Los nuevos modelos empresariales se articulan en base a estructuras horizontales donde la innovación y el estímulo a la creatividad son esenciales. Éstos promueven el desarrollo integral del individuo, así como su identidad, compromiso y lealtad para con la organización.

La empresa moderna exige que sus trabajadores sean multifuncionales, que estén preparados para las contingencias y que puedan y sepan trabajar en grupo; además las organizaciones se comprometen a una permanente calificación del personal, a una delegación de responsabilidades y a crear un liderazgo compartido.

La actividad turística no está exenta de cambios y demandas a nivel de las organizaciones empresariales; de allí la necesidad de adecuarse y ser flexible en una actividad dinámica y extremadamente competitiva.

La industria del turismo está en una constante evolución desde la década del 60 hasta el presente, que ha marcado cambios y preferencias por parte de los consumidores y por parte de la oferta 
de servicios que han tenido que adecuarse a las necesidades de los primeros. La evolución en el comportamiento y valores de los consumidores es la principal fuerza motora del nuevo turismo. Se busca tener una mayor experiencia en viajes, una mayor flexibilidad en las facilidades, considerando el sentido de independencia que sienten los nuevos turistas y que por tanto, generan una demanda de mayor calidad.

Los cambios que presentan los consumidores reflejan, sin lugar a dudas, la situación demográfica, de transporte, de condiciones económicas, y de costumbres de los mismos. Los cambios en el estilo de vida del nuevo turista presentan una demanda por vacaciones más personalizadas, lo cual produce el surgimiento de una nueva oferta diferenciada y de calidad.

Por otro lado, los proveedores de servicios turísticos están beneficiándose de los cambios de la vida moderna y del uso de la tecnología que facilita la estructura y venta del producto. Los avances en informática y comunicación han revolucionado el mundo y el turismo lo está aprovechando. Hoy en día conocer, informarse, seleccionar y tomar la decisión de compra de un producto turístico, es muy sencillo; sin embargo, ello implica que el consumidor esté habilitado en el uso de la tecnología y que el productor del servicio turístico sepa utilizar los canales de comercialización más adecuados de acuerdo a cada tipo y segmento de mercado.

Adicionalmente a ello la liberalización del transporte aéreo, la liberalización del comercio de servicios y la integración económica producida entre diversos países ha traído como consecuencia una mayor posibilidad de desplazarse por el mundo por motivos de turismo, con facilidades logísticas que permiten conocer y disfrutar de nuevos destinos y para ello también el consumidor tiene que estar calificado, para aprovechar de la mejor manera los medios que le proveen de la información necesaria para el viaje. Nuevamente el recurso humano debe ser capaz de utilizar la tecnología para su satisfacción personal en materia de viajes.

No debe olvidarse que el trabajador es el eje fundamental y la razón de ser de la organización, por ello las empresas con visión y perspectivas de futuro dirigen su atención al desarrollo y mejora del talento humano. Esto se convierte en una inversión que será altamente rentable en la medida que la empresa mantenga una buena relación con el trabajador y en tanto el trabajador se identifique con la empresa.

Con la invención de la computadora en la década del 50 comenzó la era de la información que se sustenta en el avance tecnológico, principalmente en los medios de comunicación, pero también obligó a que los trabajadores se capacitaran y entendieran los cambios que se generarían producto de la incorporación de las maquinas inteligentes al trabajo y, por consiguiente, de su participación en él.

De otro lado, las nuevas técnicas administrativas sustentadas en la aplicación de las ciencias sociales, la psicología, y la sociología permiten conocer el funcionamiento de la sociedad, la actuación de los individuos en ella y las relaciones que se dan entre dichas ciencias: si la psicología se centra en la conducta de los individuos y en los motivos que los impulsan a comportarse de una forma terminada, la sociología se centra en la interacción de dos o más individuos que forman parte de dos grupos y que conforman el colectivo. De la confluencia de la participación de estas ciencias se generó la forma de entender y de plantear la productividad en el trabajo.

Las tendencias organizacionales actuales responden, sin lugar a dudas, a las motivaciones y comportamiento de la demanda y ésta última cada vez es más dinámica, de ahí la necesidad de que el trabajador esté adecuado a esos cambios de tipo operativos y mentales, y con una predisposición definida para ello.

La administración actual ha variado sus niveles de relación interna en la empresa. de la tradicional relación vertical de jefe a subordinado que era rígida y cerrada, se ha pasado a una relación jerárquica horizontal, flexible, abierta y con mayores beneficios. Entonces, si el empleado siente que es un eslabón importante en la cadena laboral y que tiene la posibilidad de expresar su opinión y emitir sugerencias, su productividad será mayor y su sentido de pertenencia y lealtad para con la empresa se incrementará, más aún: potenciará, por propia iniciativa, sus destrezas y habilidades para la creación del producto.

Si al ingresar a una organización empresarial el trabajador está identificado con ella y tiene conocimiento de su vida institucional o empresarial, de los logros, productos, de su posición en el mercado, de las características de la demanda 
del producto, entre otros, éste podrá desarrollar una cultura empresarial que desembocará en la identidad para con la empresa y en productividad para con su empleo.

Es importante reconocer que en materia de turismo, en términos generales, no se habla de gran empresa sino que, por el contrario, los verdaderos forjadores de la actividad turística son las pequeñas y medianas empresas. Éstas se ven obligadas a hacer frente a los efectos de la mundialización y deben adaptarse a las nuevas condiciones del mercado, por lo que tienen que desarrollar estrategias atractivas, exitosas y diferentes en la formulación de los productos turísticos, en el posicionamiento de su empresa en el mercado y en el posicionamiento de su marca.

Para que las pequeñas y medianas empresas turísticas puedan competir y ser exitosas tienen que instalar nuevos sistemas y, fundamentalmente, formar a su personal en el uso de las tecnologías relacionadas al turismo. Sin embargo, ello supone la necesidad de invertir en actividades de formación y capacitación del personal y es ahí donde hay restricciones que en muchos casos suponen diferir la acción misma, lo que perjudica a la calidad del servicio ofrecido por la empresa y su situación competitiva en el mercado.

Si las pequeñas y medianas empresas dependen de su disponibilidad financiera, de su acceso al mercado, y del acceso y uso de los canales de distribución, entonces tienen que centrarse en la capacitación de su personal de tal manera que signifique una fortaleza para la empresa.

Este tipo de empresas -que ser la mayoría en el caso del turismo- tienen que desarrollar una política de personal basada en las competencias, calificaciones y roles que tienen que desempeñar para lograr un beneficio doble; es decir, para la empresa y para sí mismo como persona.

Según la OMT (2001), las pequeñas y medianas empresas son un agente importante de creación de empleo aunque con bajos niveles de productividad y de calidad de los productos, así como por la falta de acceso al crédito y a la formación profesional.

Es clara la fuerza productiva que suponen las pequeñas y medianas empresas aunque también son claras las restricciones y limitaciones que tienen en cuanto a la calificación y profesionalización de su masa laboral: respecto al empleado, las restricciones se presentan en aceptar su rol, funciones, atribuciones, y cambios de puestos que se generan en las empresas por el dinamismo imperante; en cuanto a las empresas, los problemas se presentan para adecuarse a la nuevos productos y a su reingeniería cuando es necesario.

El trabajador actual ha cambiado. Tiene una mayor preocupación por estar más calificado, considerando lo exigente del mercado y la gran presencia de posibles nuevos trabajadores para su puesto.

En definitiva, hay que reconocer que el centro neurálgico de una empresa son sus trabajadores, quienes deben estar capacitados para la función que desempeñan y la empresa tiene que diseñar políticas especiales de recursos humanos.

La especialización y las diferencias cualitativas entre las posibles ofertas turísticas deben aportar un valor agregado y de calidad que, en la mayor parte de las ocasiones, vendrá aparejado los recursos intangibles. Uno de los recursos de carácter intangible que incide de forma directa en la diferenciación del producto en la actividad turística es el capital humano. Se abre así una carrera hacia la competitividad en la cual las reglas de juego están continuamente cambiando, de tal manera que es en la especialización de propuestas y en los intangibles donde es necesario buscar una ventaja competitiva de carácter sostenible.

El sector turismo, dadas sus características y naturaleza, presenta limitaciones para atraer y mantener a trabajadores calificados, así como para mejorar las calificaciones de los que ingresan al mercado laboral, para estabilizar la fuerza de trabajo e incrementar la productividad de las empresas y la calidad de los servicios.

La diversidad de empresas que conforman la estructura productiva de la actividad turística demanda diversos tipos de conocimientos por parte de los trabajadores. Sin lugar a dudas, los servicios de alojamiento y de alimentación constituyen -en mayor proporción- los servicios turísticos, por tanto los recursos humanos que en ellos se desenvuelven deben tener altos niveles de calificación y estar preparados y permanentemente alertas ante los cambios que se presentan en su actividad.

Por otra parte existe un factor que tiene un peso psicológico muy importante en el trabajador, que es la estabilidad en el empleo. Un trabajador 
desempeñará mejor sus funciones si sabe que es estable y que su mejora jerárquica y remunerativa depende en gran medida de su desempeño laboral.

La actividad turística, por su propia naturaleza y por la estacionalidad que la caracteriza, presenta una elevada rotación de personal. Esta rotación debería atribuirse al carácter temporal de la fuerza de trabajo así como a la dificultad general para conservar al personal. Los trabajadores mencionan que los bajos salarios son una de las causales de esta constante rotación, aunque la falta de una carrera profesional y prestaciones parecería tener más importancia. En empresas donde la masa laboral es activa, está motivada y recibe la formación necesaria para desarrollar sus funciones, se observa que la tasa de rotación es inferior.

En la medida que las empresas se adecúen al mercado, potencien su organización interna, fortalezcan y amplíen sus redes de comercialización, y cuenten con personal motivado y calificado mediante cualquiera de los niveles de formación o capacitación, su competitividad será mayor y podrá desarrollarse adecuadamente en el mercado.

La competitividad obliga a desarrollar nuevas formas de gestión y manejo de los recursos humanos, los cuales demandan una mayor especialización producto del constante cambio del sector pero, a su vez, deben tener valores éticos que les permitan estar comprometidos y ser leales con la empresa que los contrata.

En relación a la administración de empresas, Wikipedia ${ }^{1}$ denomina recursos humanos al trabajo que aporta el conjunto de los empleados o colaboradores de una organización. Lo común es denominar recursos humanos a las áreas que tienen a su cargo la función de seleccionar, contratar, formar, emplear y retener a los colaboradores de una organización.

Las empresas turísticas tienen la obligación de desarrollar programas de manejo de los recursos humanos los cuales deberán iniciarse con:

- Definición de la marca del empleador. Es fundamental que la empresa posea una marca definida, la cual deberá ser internalizada por el trabajador y asimilada de tal manera que el compromiso empresarial no suponga una obligación sino una manera "natural" de asumir las cosas. La marca del empleador posibilita el compromiso y lealtad para con la empresa frente a empresas de similar rubro que podrían ofrecer mejores condiciones al trabajador.

- Mecanismos de comunicación interna. Es indispensable el establecimiento de líneas de comunicación y coordinación entre los niveles jerárquicos o ejecutivos, los medios y los operativos; pero a su vez es necesario el establecimiento de mecanismos de carácter transversal que faciliten la comunicación dentro de los mismos niveles. La comunicación interna es un factor primordial para que la información sobre los productos que genera la empresa fluya de tal manera que toda la cadena de valor esté comunicada.

- Mecanismos de comunicación externa. La empresa debe establecer los mecanismos de comunicación pertinentes con sus empresas proveedoras, con sus empresas compradoras y con aquellas empresas e instituciones con las que se relaciona directa o indirectamente. Los medios a utilizar dependerán del tipo de comunicación y del segmento al cual se dirijan.

De otra parte las organizaciones empresariales deben contar con áreas especializadas en el manejo de los recursos humanos, por cuanto hay que destacar:

- Estrategia de recursos humanos. La empresa deberá establecer la estrategia a seguir con el personal de la empresa; es decir, cómo dirigirlos respetando sus derechos personales.

- Convocatoria reclutamiento y selección. La empresa deberá establecer los parámetros a considerar para el proceso de convocatoria, selección y capacitación del personal; ello permitirá contar con personal idóneo en cada puesto ocupacional.

- Formación. En función a los puestos ocupacionales, la empresa deberá establecer un profesiograma de los mismos y definir la formación que debe tener cada empleado.

- Evaluación del desempeño. En base a criterios técnicos y racionales se deberá evaluar periódicamente a los trabajadores con la finalidad de conocer su rendimiento, su perspectiva y opinión con respecto a su función como trabajador y a su relación con el producto y con los vendedores del mismo.

1 Información obtenida de Wikipedia (2009). 
- Relaciones con los empleados. Siendo el trabajador el eje principal de la empresa, es sustancial que se establezcan las líneas de coordinación internas que permitan conocer el grado de empatía entre los trabajadores y el grado de productividad con relación a esa empatía.

Es importante destacar que la política de recursos humanos cubre todo el proceso del personal; sin embargo, hay que mencionar que es imprescindible la buena voluntad y predisposición del trabajador para que ésta sea productiva. Un factor primordial en las relaciones humanas es, sin lugar a dudas, la motivación que, según Dalton², es el estímulo emocional que nos hace actuar; es decir, se trata de un impulso que condiciona las conductas. En el ámbito laboral la empatía es fundamental para el desarrollo de las funciones de la empresa y su empatía con todos los trabajadores.

Actualmente las organizaciones inciden fundamentalmente en la motivación como una estrategia fundamental para el desarrollo de los recursos humanos. El modelo básico de conducta surge de la necesidad, lo que genera tensiones en el individuo que le impulsan a realizar acciones tendientes a solucionar dicha necesidad, para lo cual pone en juego sus habilidades y destrezas. La solución de esa necesidad genera una sensación de satisfacción y de alivio que permite sentirse mejor.

Al ser el turismo una actividad de servicios, es decir perteneciente al sector terciario, Clark${ }^{3}$ demanda una variedad de empleos calificados que permitan satisfacer las necesidades de los visitantes. Es por eso que el personal que labora en el sector tiene que estar permanentemente calificado y eso es responsabilidad del empresario, por cuanto a mayor calificación del personal, mejor calidad en el servicio y por tanto mayor productividad en la empresa, y mayor presencia y participación en el mercado. La actividad turística en esta época de modernidad tiene que ser competitiva, vale decir, debe ser capaz de proveer el servicio de manera adecuada y oportuna, considerando que el mercado turístico es muy dinámico.

Otro aspecto importante a reconocer es que en la actividad turística se dan altos niveles de rotación de empleo; ello debido -en parte- a la necesidad del trabajador de mejorar en su puesto ocupacional, así como de mejorar su calidad de vida. Es tradicional que la empresa capacite a su personal y que éste, una vez que está mejor calificado, migre a otra empresa por motivos económicos o de posición ocupacional.

La actividad turística está caracterizada por concentrar la mayor fuerza laboral en restaurantes y establecimientos de hospedaje donde, tradicionalmente, ha sido el género masculino el predominante, aunque en los últimos años se ha ido incorporando el género femenino gradualmente.

También es importante destacar la incorporación paulatina aunque no siempre a tiempo completo de una masa laboral joven, principalmente estudiantes que inician sus labores como practicantes y van ascendiendo en la empresa. Cabe anotar que en muchos casos se da una rotación muy fuerte, teniendo en cuenta que el estudiante está en plena búsqueda de un puesto de trabajo más acorde con sus expectativas.

Así podría caracterizarse al empleo en el sector turismo de la siguiente manera:

- Concentrado en un $70 \%$ en la actividad hotelera y de restaurantes.

- Concentrado en un $60 \%$ en el sexo masculino.

- Empleos con contratación temporal del tipo locación de servicios (de tres a seis meses), sin mayores beneficios sociales.

- Gran porcentaje de empleados sin preparación académica, con experiencia obtenida en el trabajo, empíricos.

- Incorporación paulatina y gradual de egresados universitarios.

- Concentrado mayoritariamente en pequeñas y medianas empresas.

- Organización empresarial prioritariamente de carácter familiar.

En consecuencia, el manejo y gestión de los recursos humanos en una empresa o institución es fundamental para crear, mantener y consolidar la imagen empresarial y del producto en la mente del trabajador, así como reafirmar su lealtad hacia la empresa.

En el caso del turismo, al ser una actividad con diversos componentes y ser -fundamentalmente- un servicio, la labor tiene que ser constante y enfática para posicionar la empresa en la mente

2 Dalton. (2007).

3 Clark. (1978). 
del trabajador. Es importante destacar que según Gallego ${ }^{4}$, el activo más importante de la empresa es la cantidad y calidad de conocimientos que dispone su personal y cómo lo utilizan. Este conocimiento es aprovechado y aplicado en las funciones por el personal que labora en la empresa; de la suma de las capacidades individuales y de la sinergia de los grupos que laboran en la empresa se genera el verdadero valor para competir en los mercados cada vez más amplios, diversificados y exigentes. Los diversos modelos de formación y capacitación, es decir de calificación de los recursos humanos, tienen un objetivo común que es la eficiencia.

El personal de una empresa tiene tareas y funciones asignadas, tiene medios y herramientas de soporte para sus habilidades, y tiene un sólo derrotero: brindar un producto de calidad para lograr un consumidor satisfecho. Por otro lado, más allá de la aplicación del conocimiento y del uso de las técnicas y herramientas, subyace un aspecto medular que es la cultura organizacional. Ésta debe generar entusiasmo, inducir a la creatividad y a la innovación, buscar simplificar los procesos para hacerlos más eficientes, y redireccionar los profesiogramas de los puestos ocupacionales, buscando una mayor coincidencia entre la oferta y la demanda laboral.

En definitiva, el manejo de los recursos humanos tiene que ser direccionado en función a los objetivos de la empresa y al tipo de producto que ésta produce, teniendo en cuenta bajo todo punto de vista su posición en el mercado.

\section{TURISMO Y EMPLEO}

Al estar compuesta la actividad turística por diversos elementos muy diversificados, el empleo se presenta de igual manera y, por lo tanto, su estudio y análisis se torna complejo.

Diversas organizaciones internacionales como la Organización Internacional del Trabajo (OIT) y la Organización Mundial del Turismo, y en el ámbito nacional instituciones como el Ministerio de Comercio Exterior y Turismo (MINCETUR), PROMPERÚ y el Ministerio del Trabajo y Promoción del Empleo (MTPE), han realizado diversos estudios sobre el tema, que permiten esbozar el perfil del empleo en el sector turismo.
El turismo es una de las actividades que presenta gran movimiento no sólo económico sino también en la generación de empleo a nivel mundial; así la OIT $^{5}$ consideraba que:

- El turismo concentraba a 200 millones de trabajadores aproximadamente, lo que representaba el $8 \%$ del empleo a nivel mundial.

- El $70 \%$ de la fuerza laboral estaba constituido por mujeres.

- El 50\% de los trabajadores tiene 25 años o menos.

- Un empleo directo en este sector da lugar a 1.5 empleos indirectos.

La Oficina Internacional del Trabajo (OIT) ${ }^{6}$ sostiene que

"El concepto económico de turismo abarca los viajes personales (por ocio u otras razones) así como los viajes de negocios, tanto al extranjero como al interior del país. Los servicios directos o cara a cara que la industria ofrece a los turistas representan entre el 3\% y $4 \%$ del PBI de la mayor parte de la economía mundial y proporcionan empleo a cerca del $3 \%$ de la mano de obra del planeta, aunque algunos países emplean hasta un $10 \%$ de la fuerza de trabajo. Actualmente la industria crece en todo el mundo a un ritmo cercano al $3 \%$ anual. Europa presenta un índice de crecimiento inferior a la media de $2.3 \%$, y Asia y el Pacífico tienen el índice más bajo (1.4\%), tras la crisis asiática. Últimamente, los índices de crecimiento más elevados han correspondido al sur de Asia (9.1\%), El Caribe (6.8\%) y Europa Central y Oriental (5.2\%)".

Mediante este informe la OIT muestra el crecimiento sostenido del sector turismo y la capacidad de generar empleo directo a nivel mundial. Ante este panorama podemos afirmar la importancia de la industria sin chimeneas como vehículo capaz de generar empleo en establecimientos de primera línea.

Teniendo en consideración las motivaciones de viaje (por ocio u otras razones), el país muestra una oferta turística diversificada, lo cual permite ir en el mismo sentido de la corriente del crecimiento turístico mundial, según concluye el mencionado informe. 
"La educación y la formación en materia de turismo se encuentran a cargo de toda una serie de instituciones, algunas públicas, otras semipúblicas (como las asociaciones comerciales) y otras incluso de propiedad privada. Los sistemas de formación a distancia de divulgación mundial que utilizan CD ROM y tecnología Internet interactiva comienzan a funcionar y están alterando profundamente el negocio de la formación, aumentando la calidad y disminuyendo los costos pese a las enormes inversiones que se están realizando en los nuevos instrumentos de formación".

En el Perú se puede apreciar un mayor compromiso por parte de las universidades, para estar acordes con la necesidad de crear escuelas profesionales para la enseñanza de turismo y hotelería. En 1980 se inició en Lima la enseñanza universitaria en turismo y hotelería; con el transcurrir del tiempo se han ido incorporando institutos tecnológicos y centros de capacitación.

Siendo España un destino turístico por excelencia en cuanto a las llegadas internacionales y el turismo interno, Albarracín ${ }^{7}$ manifiesta que

"en cualquier caso, el pilar del empleo es una base poco atendida. A pesar de haber crecido el empleo hasta ahora, no obstante, el crecimiento de las pernoctaciones $y$, sobre todo, la presencia de turistas han crecido en mucha mayor medida. Se puede decir que ni se crea todo el empleo que se requiere ni se emplea de manera estable. En suma, el crecimiento económico se ha hecho a costa de los trabajadores en todos estos años, que han padecido una nefasta gestión de la estacionalidad, y poco se ha invertido en su futuro lo suficiente".

Así muestra que el crecimiento turístico en España no está orientado a satisfacer las necesidades de los trabajadores ya que el tipo de empleo que se da en la sociedad turística española no es el adecuado, porque no existe una buena gestión y tampoco se asegura un retiro digno a sus trabajadores una vez que se jubilen.

El Consejo Mundial de Viajes y Turismo, y la Fundación Pro-Educación en Turismo indican que existe una

"escasez de empleados, ya que más de un tercio de los empresarios encuestados han tenido

7 Albarracín. (2004)

8 Torruco. (2009).

9 SERNATUR. (1999). dificultades para reclutar directivos calificados, y más del 40\% ha tenido dificultades para reclutar personal capacitado o parcialmente capacitado".

En esta publicación los empresarios mexicanos, a pesar de que su país es una potencia turística mundial, muestran una preocupante falta de profesionales especializados en turismo para desempeñar cargos de alta responsabilidad. También es una realidad lo difícil que resulta asimilar trabajadores capacitados y peor aún poco capacitados. Ante esta realidad los mexicanos tienen que hacer mayores esfuerzos para formar cuadros directivos y capacitar al personal que va a laborar en la actividad turística.

La percepción de la industria turística es que ésta tiene un estatus modesto dentro de la sociedad mexicana. Una gran proporción de empresarios, así como una pequeña cantidad de funcionarios, perciben que la industria recibe poco reconocimiento y apoyo por parte de los gobiernos federal y estatales. A pesar de la importancia de la actividad turística en México, ésta no es bien vista, lo que impide que las nuevas generaciones de mexicanos vean el turismo como una buena alternativa para el desarrollo profesional.

En cuanto a la educación turística, Torruco ${ }^{8}$ menciona que la profesionalización del sector reviste gran importancia para lograr mejores servicios y una calidad competitiva a nivel internacional. Menciona que si se están construyendo 22,500 habitaciones anuales y “... cada habitación genera al país un empleo directo y tres indirectos”, es indispensable la profesionalización en todos los niveles ocupacionales.

Enfatiza que la formación en turismo es una necesidad que no se puede postergar y que en México, de cada 100 alumnos que egresan de carreras turísticas, 80 tienen un empleo remunerado y de éstos, 20 están en el sector turístico. Merece destacarse la necesidad de establecer una estrecha relación entre los Ministerios de Educación, Turismo y Trabajo, con el sector privado para lograr la profesionalización del sector turístico.

Por otra parte, en un estudio realizado en Chile por SERNATUR ${ }^{9}$ se sostiene la

“... necesidad de contar con recursos humanos especializados. Los requerimientos de recursos 
humanos planteados se concentran de manera mayoritaria en la actividad restaurantes y similares (52\% del total). En esta actividad, las ocupaciones más solicitadas se refieren a garzones (mozos) y cocineros. En la actividad de comercio turístico ( $10 \%$ de los requerimientos), las solicitudes se refieren principalmente a vendedores y recepcionistas. De igual manera los hoteles y otros tipos de alojamiento temporal y el transporte de pasajeros por vía terrestre señalan importantes requerimientos. Los primeros, en las especialidades de mucama, aquellas relacionadas con la cocina y personal administrativo; y en el segundo, conductores".

Este estudio muestra la necesidad mayoritaria de personal, principalmente de mozos y cocineros. Se puede destacar que un alto porcentaje de ese tipo de requerimientos se está cubriendo con la migración de trabajadores peruanos que viajan a Chile y con la presencia de empresarios nacionales que están invirtiendo en negocios de restaurantes.

En nuestro país tenemos a PROMPERU ${ }^{10}$ que afirma que:

"La demanda turística futura muestra creciente interés hacia el ecoturismo y una relativa pérdida de interés en el turismo históricocultural que, es actualmente, el que capta más turistas hacia el Perú. Si bien las cifras indican que para el 2005, el turismo histórico-cultural seguirá siendo el más atractivo en el Perú con 92\% de menciones, y el ecoturismo ocuparía el segundo lugar con 48\%, para el 2021 a ambos se les otorga el $64 \%$ de interés".

Este estudio muestra hacia qué tipo de turismo debe prepararse el Perú para el futuro; si bien es cierto que actualmente estamos posicionados internacionalmente como un destino históricocultural, la tendencia indica que para el 2021 el ecoturismo habrá crecido a niveles similares, lo que podemos identificar como una señal de alerta para formar profesionales especializados en turismo sostenible, porque cada vez existe una mayor conciencia ambiental, no sólo en los países desarrollados sino en las naciones en vías de desarrollo.

En el presente se observa que la situación no ha sufrido mayores modificaciones: la formación universitaria y no universitaria siguen siendo si- milares, y solo dos universidades han incorporado el ecoturismo como parte del plan de estudios.

"La principal crítica que realizan los entrevistados sobre los centros de instrucción es que éstos no presentan una relación adecuada entre la práctica y la teoría, además de la falta de especialización. Los institutos por los que mostraron mayor preferencia los entrevistados fueron CENFOTUR y Montemar; entre las universidades destacan San Ignacio de Loyola y San Martín de Porres".

En el informe, PROMPERÚ considera que más de la mitad de las personas que trabajan actualmente en el sector no han estudiado una carrera relacionada con el turismo, lo que repercute en la calidad de la prestación de los servicios turísticos, aunque esta problemática es responsabilidad de los prestadores de servicios turísticos, así como de las instituciones gremiales y estatales directamente involucradas en la formación y capacitación de personal.

Por otra parte, se establece que el nivel de instrucción académica es mayoritariamente técnico en un $68 \%$. Esto concuerda con la excesiva presencia de Institutos Superiores Tecnológicos y CETPROS, los cuales brindan formación técnica básicamente en hotelería, gastronomía, turismo, guía oficial de turismo y aviación, aunque muchos de ellos no cuentan con el soporte académico adecuado como plana docente, infraestructura y equipamiento académico ad hoc, y relaciones interinstitucionales adecuadas.

Es importante destacar que el estudio determinó que los mandos medio son los más requeridos por las empresas de servicios turísticos, principalmente en agencias de viajes con un $83 \%$. A estos puestos acceden egresados formados en Institutos Superiores, CETPROS y universidades; es decir, convergen de todo tipo de institución educativa. La selección del trabajador por parte de la empresa interesada tiene en consideración, fundamentalmente, la capacidad y la habilidad para el desarrollo de las funciones y atribuciones propias del puesto ocupacional, más allá de la institución educativa de formación.

En el estudio elaborado por PROMPERÚ se muestran las deficiencias de la formación profesional:

10 PROMPERÚ. (2001). 
- El contenido de los planes de estudios no guarda relación con la realidad turística.

- Se enseñan generalidades y la especialización es insuficiente.

- Los dueños de los institutos priorizan el interés económico.

- No cuentan con docentes especializados en turismo.

- Ausencia de bibliotecas.

- Falta de equipamiento.

- Infraestructura y/o locales inapropiados.

- No mantienen suficiente relación entre la práctica y la teoría.

Es importante señalar que entre las condiciones que debe cumplir el futuro profesional en turismo, además de conocimiento y dominio de las funciones y tareas del puesto ocupacional, se establece:

- Dominio de idiomas.

- Conocimiento sobre la realidad y el turismo en el país.

- Dominio de técnicas gerenciales.

- Mayor dinamismo, creatividad e iniciativa.

Merece especial atención la Universidad de San Martín de Porres, pionera en la formación de profesionales en turismo y hotelería, la que mantiene su liderazgo dentro y fuera del país, siendo considerada por la Organización Mundial del Turismo como una de las trece instituciones educativas en turismo más importantes del mundo.

Es necesario evidenciar las restricciones que se presentan en la generación del empleo. Así tenemos que Chacaltana ${ }^{11}$ señala que:

“... al margen del turismo realizado por nacionales fuera del país, existen dos tipos de turistas: los nacionales y los extranjeros. En países desarrollados, el turismo interno es el más activo. En Perú la situación es la inversa, aún cuando PROMPERU estima que existen 1.6 millones de turistas internos al año, cifra superior a los 900,000 turistas extranjeros, lo real es que existen abismales diferencias entre ambos tipos de turistas. Para empezar, el nivel de gasto entre uno y otro grupo es totalmente diferente. Mientras los turistas extranjeros tienen niveles de gasto medio diario de alrededor de US\$ 120, los nacionales gastan menos de US\$20. Esto se debe evidentemente al mayor poder adquisitivo de sus países de origen (Estados Unidos, Francia, Inglaterra) que es bastante más elevado Pero también tienen características individuales diferentes: una educación largamente superior, mayor edad y condiciones de trabajo más ventajosas. Todas estas diferencias influyen en el impacto que generan los diferentes tipos de turistas, tanto en la economía como en el empleo en el Perú. Mientras los turistas nacionales utilizan como hospedaje las residencias de familiares o amigos, los turistas extranjeros utilizan hoteles, usualmente de alta calidad. Asimismo, mientras los turistas extranjeros tienen una estancia media de 15 días, los nacionales tienen una estancia media de 8 días. Ambos tipos de turistas enfrentan restricciones de diversos tipos para la optimización de su consumo; estas suelen ser de tiempo, recursos e información. En el caso del turismo interno, el gobierno ha venido trabajando desde hace varios años bajo el supuesto de que la restricción principal es de tiempo. En países desarrollados ésta suele ser una restricción importante, especialmente debido a la alta tasa de empleo existente. En países como el nuestro, la restricción principal parecería ser de ingresos. Los bajos ingresos de los consumidores peruanos limitan fuertemente el desarrollo del turismo interno, pero esta es una variable que sólo puede ser afectada a través de una política económica y no sectorial. Lo que se puede trabajar a través de la política sectorial es la difusión de información de diverso tipo, desde alternativas de destino hasta modalidades y facilidades para el traslado y hospedaje. Ésta es una de las mayores deficiencias actuales en la política pública de promoción del turismo en el país. Las fallas del mercado que aparecen en este sector se derivan de la naturaleza del bien o servicio que se comercializa. Como se puede intuir, el turismo es un bien compuesto, cuyo proveedor no es uno solo sino muchos, algunos de los cuales incluso podrían no estar de acuerdo en definir a su producto como turístico. La diversidad de empresas involucradas en este sector difiere no sólo en tecnología y calidad sino también en objetivos económicos. En

11 Chacaltana. (2002). 
todo caso, la naturaleza del producto turístico implica la presencia de fallas del mercado que limitan la posibilidad de soluciones privadas óptimas en el sector".

En primer lugar, existe una fuerte presencia de bienes públicos. Los productos turísticos peruanos (y de muchas partes del mundo), en su mayoría, se construyen sobre la base de algún bien de naturaleza pública, lo cual hace imposible que el sector privado por sí sólo pueda encontrar soluciones eficientes desde el punto de vista individual y social. Por tanto es necesaria la intervención del Estado, especialmente en otros bienes públicos que faciliten la comercialización del atractivo público, como la inversión en infraestructura vial, información entre otros. Los bienes públicos pueden financiarse vía la inversión directa del Estado o vía concesiones, que es un esquema que parece será utilizado con más fuerza en el futuro.

En segundo lugar, en este sector existen también numerosas externalidades tanto positivas como negativas. Cualquier operador turístico privado sabe que la satisfacción del cliente, por ejemplo, no depende sólo de sus esfuerzos, sino de cómo otros proveedores públicos o privados traten al turista. Por más que un empresario turístico se esfuerce en mejorar, si falla una parte de la cadena de servicios que conforman el producto turístico, toda la calidad del turismo se verá comprometida. Esto requiere de un gran esfuerzo de cooperación y coordinación entre proveedores privados y entre éstos y el Estado. Usualmente es el Estado o los gremios de proveedores turísticos los que deben velar porque estas externalidades puedan ser internalizadas.

Entre los estudios sobre las perspectivas de crecimiento y generación de empleo en turismo en el Perú, Chacaltana ${ }^{12}$ sostiene que:

"Tanto en este sector (hotelero) como en el de transporte, la oferta que más rápido ha reaccionado es la asociada a los capitales multinacionales. Esto permite atender un mayor número de turistas en el corto plazo pero a largo plazo su principal desventaja es que reduce el nivel de los multiplicadores de ingresos y de empleo. Si las filtraciones llegan a ser muy altas, se pueden comprometer los efectos beneficiosos que se suele atribuir al desarrollo del turismo. En otras palabras, es importante realizar un exhaustivo seguimiento del tipo de crecimiento que tenga este sector en los próximos años".

Es importante destacar que la inversión extranjera tiene limitaciones porque, por ejemplo, el directivo de un establecimiento de hospedaje, además de sus honorarios profesionales, recibe beneficios adicionales como alojamiento, alimentación, bebidas, llamadas telefónicas, lavandería; por lo tanto remite a su país de origen remesas mensuales que no se filtran a la economía local. Los trabajadores locales ocupan puestos de mando medio y de servicios obteniendo menores sueldos que los extranjeros.

"En el mejor de los casos, hacia el año 2010 se podría alcanzar la cifra de 2.5 millones de turistas, lo cual implicaría la generación de aproximadamente 1,390 millones de nuevos empleos en el sector. En ese escenario general es importante la información para que los jóvenes vean esta actividad como una alternativa genuina para su desarrollo profesional y los incentivos adecuados para que las empresas del sector inviertan en la formación de su personal".

Esta proyección al año 2010 es importante porque genera nuevas expectativas para los futuros profesionales en turismo, más aun considerando el comportamiento de crecimiento sostenido que han tenido las llegadas internacionales hasta el 2008, aún habiéndose presentado dificultades de recesión económica y de salud a nivel mundial.

En este esfuerzo por conocer y analizar la situación del empleo en el país, Marsano ${ }^{13}$ considera que:

"A través del estudio se ha podido verificar que en los establecimientos de hospedaje de 5 estrellas existe un promedio de 49\% de egresados de universidades que cuentan con la carrera de turismo y hotelería, siendo este el mayor promedio. Esto indica que en los hoteles de mayor categoría existe una mayor conciencia de la importancia de contar con profesionales formados para la actividad turística".

Se puede apreciar que en los establecimientos de mayor categoría se da una mayor exigencia por parte de los clientes y la competencia es más fuerte. Para que éstos se mantengan en un nivel

12 Chacaltana. (1999).

13 Marsano. (2002). 
óptimo de competitividad es necesario contar con profesionales especializados en turismo y hotelería.

En esa búsqueda por conocer la situación del empleo, el Ministerio de Trabajo y Promoción del Empleo ${ }^{14}$ efectúo una Encuesta sobre Calificación de los Trabajadores y sus Competencias Laborales en el Sector Turismo. El Estudio resalta la importancia de la recuperación y el crecimiento del sector turismo y precisa que es necesario contar con el capital humano necesario para los requerimientos del creciente mercado. Reconoce también la existencia de un desajuste entre la formación de la carrera profesional y los requerimientos ocupacionales, lo que evidencia una brecha que se observa en la calidad de la prestación del servicio.

Precisa que el sector turismo requiere de gran mano de obra concentrada prioritariamente en los restaurantes y el transporte, y que esto tiene implicancia a nivel nacional por las propias características de la actividad, eminentemente descentralizadora. Así considera que el $41 \%$ de los empleos corresponde a restaurantes, el $36 \%$ a transporte y el $11 \%$ a esparcimiento, los que en su conjunto representan el $88 \%$ de la generación de empleo. Esto evidencia nuevamente el tipo de concentración empresarial.

Fija también las remuneraciones promedio de los trabajadores de lo que se infiere que a mayor tamaño de la empresa, mayores los montos remunerativos y que éstos se concentran en los cargos de mayor responsabilidad. Cabe destacar que similar comportamiento se observa en las pequeñas empresas.

La encuesta también permite apreciar la concentración del volumen de trabajadores por el tipo de empresas: el área de cocina reúne el $24.1 \%$ del total, seguido del área de operaciones con el $17.6 \%$.

Ante el crecimiento del sector turismo es innegable que las empresas en funcionamiento así como aquellas que se incorporarán al mismo demandarán una mayor cantidad de personal, principalmente en el nivel operativo, y en menor proporción en el mando medio y en el nivel ejecutivo. Siendo complejo el conseguir personal calificado para el sector, la oferta de servicios educativos deberá contrastar su programa de formación y capacitación con los requerimientos del mercado laboral.

El Plan Estratégico Nacional de Turismo del Perú 2008-2018 (PENTUR) ${ }^{15}$ establece que, según la Cuenta Satélite de Turismo al 2001, el empleo en el sector turismo ascendía a 251,143 empleos directos y 203,143 empleos indirectos; en su conjunto, el empleo sectorial fue de 454,568 puestos a nivel nacional. Cabe destacar que aplicando el multiplicador de 1.81 a la cifra de empleo directo se ha obtenido el total del empleo generado, incluyendo el indirecto.

Continuando con la permanente preocupación por conocer el mercado laboral de la actividad turística tenemos a Regalado y Oré ${ }^{16}$ quienes consideran que 1996 es el partida del boom turístico en el país, cuando surgen nuevas empresas que se suman a las que ya habían en operación y le van imprimiendo un mayor dinamismo al sector. Asimismo consideran que dada la complejidad de la actividad en cuanto a su naturaleza, no se define muy bien si el estudio del turismo es una ciencia, una carrera técnica o una carrera profesional; por ello se torna compleja su estructura y análisis.

De igual forma manifiestan que el turismo aun habiendo presentado un crecimiento constante y sostenido en los últimos cinco años, todavía no dispone de recursos humanos adecuados para mantener las necesidades de calidad del servicio que le permitan constituirse en una actividad altamente competitiva. También reconoce que la educación superior crece sin límites y no mantiene estándares de calidad, lo cual condiciona la calidad de la prestación del servicio.

Asimismo evidencia que no obstante efectuarse diversos estudios y encuestas y ante la existencia de una gran oferta educativa no siempre de calidad, no se puede disponer de resultados de la inserción laboral en turismo. Tal carencia no permite que las universidades e institutos superiores conozcan realmente su aporte efectivo al mundo laboral, así como su coincidencia o no con los requerimientos de los puestos ocupacionales de la actividad.

Destaca que hay un grave desbalance entre la formación y la manifiesta en dos sentidos:

- Entre la oferta de servicios educativos y la demanda laboral.

14 Ministerio de Trabajo y Promoción del Empleo. (2006).

15 Plan Estratégico Nacional de Turismo del Perú 2008-2018 (PENTUR). (2008).

16 Regalado y Oré. (2009). 
- Entre el volumen de egresados y los insertados en el mercado laboral.

En consecuencia, reconoce la importancia de la profesionalización en turismo en cuanto al empleo, lo cual va a permitir alcanzar estándares internacionales de calidad y convertir al producto turístico del país en uno altamente competitivo.

Referente a las nuevas formas de organización del Trabajo, la Organización Mundial del Turismo $(\mathrm{OMT})^{17}$ establece algunos criterios a considerar como:

- La flexibilidad del trabajo. Si bien es cierto el turismo demanda de un trabajo continuo y para el cual el trabajador debe estar consciente de cierta rigidez que significa el brindar el servicio turístico oportuno, es importante destacar la necesidad de una reducción de las prácticas rígidas para fomentar una mayor movilidad del personal, productividad e innovación. Así se han dado cambios como el reenfoque de las competencias de base, la modernización de las cualificaciones y especializaciones, la creación de nuevos perfiles de profesionales y el desarrollo motivacional necesario. Es decir, puede darse el caso de compartir una función pero definiendo los parámetros y márgenes dentro del cual cada trabajador debe actuar y asumir su responsabilidad. Por otra parte la reorientación y redefinición de los profesiogramas en las áreas de alojamiento y restauración principalmente, han dado como consecuencia una reorientación y flexibilización del trabajo.

- El empleo estacional. Considerando que en muchos destinos el turismo es estacional, quedan espacios libres entre una temporada y otra. La tendencia sería la utilización de ese tiempo libre para capacitar y especializar al personal que labora, como un periodo de preparación para la siguiente temporada. Esto significa que la empresa asuma el costo de la capacitación como una inversión que redundará en un mejor rendimiento del trabajador, una mayor calidad del servicio turístico, una mayor rentabilidad económica de la empresa, en una mejor imagen $y$, por tanto, en un mejor posicionamiento en el mercado.

- La polivalencia profesional. Este aspecto es de mucho interés para los empleadores, habida cuenta del dinamismo que presenta la actividad turística y de los costos que supone ofertar un servicio de calidad. La polivalencia profesional se considera como la posibilidad del trabajador de desempeñar diversas funciones en distintos momentos, combinando las tareas de varios trabajos en uno solo. Esta es una forma de crear o conservar un trabajo a tiempo completo en vez de varios trabajos a tiempo parcial. Sin embargo, ello demanda una identificación previa de los puestos ocupacionales a cubrir y de las características que deben tener los trabajadores para el desempeño de la función; así se podrán reducir costos al no tener que contratar especialistas adicionales a los trabajadores ya empleados. En esto juega un papel importante la ayuda de las tecnologías que facilitan la función. Un aspecto medular lo constituyen la motivación y la convicción del trabajador por el rol que va a desempeñar al ser polivalente en funciones en un área determinada.

\section{CONCLUSIONES}

La $\mathrm{OMT}^{18}$ considera que el turismo es uno de los sectores de la economía que más rápidamente genera empleo; por ende, beneficia al desarrollo de las economías emergentes.

Aun considerando la fuerte recesión económica y los fenómenos climáticos y de salud que se han presentado en los últimos años, el turismo ha seguido creciendo aunque a una tasa del $2 \%$ en el año 2008, con referencia al 2007. Se estima que en el año 2010 se alcanzaran los 400 millones de empleos en turismo, actualmente calculados en 250 millones, lo que representa aproximadamente el $11 \%$ de la PEA mundial. Asimismo un puesto de empleo directo, genera 1.5 puestos de empleo indirectos; por ello las empresas, principales centros de absorción de trabajadores, deben reconsiderar su posición frente al empleo y trascendencia.

Es bueno recordar a Saragadoy ${ }^{19}$ cuando se refería al empleo y su relación con la empresa. Así mencionaba que en el proyecto de empresa lo laboral no ha de mirarse como una carga sino como una palanca que puede ser el motor de la eficiencia de la empresa. Vale decir, el empleo es un factor catalizador de las actividades socioeco-

17 OMT. (2001).

18 OMT. (2009).

19 Saragadoy. (1998). 
nómicas y por tanto del desarrollo nacional. En ese contexto, la formación y capacitación continuas son estrategias de la empresa para lograr la eficiencia y eficacia de los recursos humanos, lo que beneficiará la imagen y posicionamiento del producto turístico.

\section{BIBLIOGRAFÍA}

\section{Libros}

ALBARRACÍN, DANIEL. (2004). La industria turística y las relaciones laborales, empleo turístico en España. Madrid, Fundación Cirem.

ALIDE y BUFETE CONSULTA. (2009). El turismo: políticas de desarrollo y potencial de inversiones para la economía de América Latina y El Caribe. Curazao, ALIDE.

ARELLANO, INVESTIGACIÓN DE MARKETING. (2009). Dimensionamiento integral del aporte de la gastronomía y la industria alimentaria peruana. Lima, DESCO.

BCP SECURITIES. (2008). Perspectivas financieras y económicas para la América Latina. Lima, PUCP.

CERTO, SAMUEL. (2003). Administración Moderna. Octava Edición. Santa Fe de Bogotá, Editorial Pearson.

CHACALTANA, JUAN. (1999). El turismo en el Perú: perspectivas de crecimiento y generación de Empleo. Lima, OIT.

CHACALTANA, JUAN. (2002a). Indicadores dinámicos del empleo. Lima, CEDEP.

CHACALTANA, JUAN. (2002b). Restricciones a la generación de empleo en el sector turístico. Lima, OIT.

CONSEJO MUNDIAL DE VIAJES Y TURISMO y LA FUNDACION PRO - EDUCACIÓN EN TURISMO. (2005). México, empleo para el milenio. México D.F., FUTUR - WTTC.

ECOGOALS CONSULTING + MANAGEMENT. (2008). Plan Nacional de Calidad Turística del Perú. Lima, MINCETUR.

FONDO MONETARIO INTERNACIONAL. (2008). Perspectivas económicas mundiales. Washington D.C., FMI.

FORO ECONOMICO MUNDIAL. (2009). Competitividad turística. Ginebra, WEF.
GALLEGO, JESÚS FELIPE. (1999). Los nuevos perfiles profesionales de los directivos hoteleros. Madrid, Editorial Alción.

HAYA DE LA TORRE DE LA ROSA, RAÚL. (2005). Estudio sobre la situación de la educación superior tecnológica en el Perú. Informe final. Lima, FORTEPE.

HERNÁNDEZ FERNÁNDEZ, BAPTISTA. (1998). Metodología de la Investigación. Mexico, Editorial McGraw Hill.

MARSANO DELGADO, JOSÉ. (2009). El impacto macroeconómico del turismo receptivo del Perú. Lima, USMP.

MARSANO MONTAVA, ANNABELLA. (2005). Desarrollo, empleo y perspectivas de los establecimientos de hospedaje de tres, cuatro y cinco estrellas de Lima Metropolitana. Lima, Tesis Maestria, USMP.

ORGANIZACIÓN INTERNACIONAL DEL TRABAJO. (2001). El desarrollo de los recursos humanos, el empleo y la mundialización en el sector de la hotelería, la restauración y el turismo. Ginebra, OIT.

ORGANIZACIÓN INTERNACIONAL DEL TRABAJO. (2009). Panorama Laboral 2008. Ginebra, OIT.

ORGANIZACIÓN MUNDIAL DEL TURISMO. (1980). Declaración de Manila sobre el Turismo Mundial. Filipinas, Organización mundial del Turismo.

ORGANIZACIÓN MUNDIAL DEL TURISMO. (1998). Introducción al Turismo. Madrid, Organización Mundial del Turismo.

ORGANIZACIÓN MUNDIAL DEL TURISMO. (1998). Los recursos humanos en turismo. Nuevo paradigma. Madrid, Organización Mundial del Turismo.

ORGANIZACIÓN MUNDIAL DEL TURISMO. (2000). Código de Ética de Turismo. Madrid, Organización Mundial del Turismo.

ORGANIZACIÓN MUNDIAL DEL TURISMO. (2001). Panorama del Turismo al 2020. Madrid, Organización Mundial del Turismo.

ORGANIZACIÓN MUNDIAL DEL TURISMO. (2008). Panorama del Turismo Internacional Edición 2008. Madrid, Organización Mundial del Turismo. 
ORGANIZACIÓN MUNDIAL DEL TURISMO. (2008). Turismo de cruceros: situación actual y tendencias. Madrid, editorial.

PACIFIC CONSULTANTS INTERNATIONAL. (1999). Plan Maestro de Desarrollo Turístico Nacional en la Republica del Perú. Fase I, Informe final. Lima, editorial.

QUISPE CALMETT, ELIANA CARMEN. (2006). La encuesta peruana al empleo y la evolución del empleo en Lima, 2001-2006. Lima, UNMSM.

REAL ACADEMIA ESPAÑOLA. (2002). Diccionario de la Real Academia. Madrid, Espasa - Calpe.

REGALADO PEZÚA, OTTO \& ORÉ BUTLER, CLAUDIA. (2009). La educación superior en turismo y las necesidades reales del mercado. Revisión de literatura. Lima, ESAN.

ROBBINS, STEPHEN. (1999). Comportamiento organizacional. Octava Edición. México D.F., Editorial Prentice Hall.

RODRÍGUEZ LÓPEZ, GREGORIO; GIL FLORES, JAVIER, Y GARCÍA, EDUARDO. (2005). Metodología de la Investigación Cualitativa. México D.F., Editorial Aljibe.

SERVICIO NACIONAL DE TURISMO. (1999). Turismo y empleo en Chile, medición, características y proyecciones. Santiago de Chile, SERNATUR.

TOFFLER, ALVIN. (1981). La tercera ola. Madrid, Cartone Editorial.

VILLENA LESCANO, CARLOS. (2006). Introducción al Turismo. Teoría y Realidad Peruana. Séptima Edición. Lima, editorial.

\section{Documentos Oficiales:}

INSTITUTO NACIONAL DE ESTADÍSTICA E INFORMÁTICA. (2009a). Encuesta permanente de empleo. Lima.

INSTITUTO NACIONAL DE ESTADÍSTICA E INFORMÁTICA. (2009b). Lineamientos en el mercado laboral de Lima Metropolitana. Informe Técnico $N^{0}$ 6. Lima.

MINISTERIO DE COMERCIO EXTERIOR Y TURISMO (2009a). Perú: Indicadores básicos para el análisis del turismo desde una perspectiva económica. Lima.

MINISTERIO DE COMERCIO EXTERIOR Y TURISMO (2009b). Perú: Indicadores trimestrales de turismo, 2007-2009, I Trimestre. Lima.

MINISTERIO DE COMERCIO EXTERIOR Y TURISMO. (2005a). Plan Estratégico Nacional de Turismo, 2005-2015. Lima.

MINISTERIO DE COMERCIO EXTERIOR Y TURISMO. (2005b). Plan Estratégico Nacional de Turismo, 2008-2018. Lima.

MINISTERIO DE ECONOMÍA Y FINANZAS. (2009). Marco Macroeconómico Multianual, 20092011. Lima.

MINISTERIO DE TRABAJO Y PROMOCIÓN DEL EMPLEO. (2007). Encuesta sobre Calificación de los Trabajadores y sus Competencias Laborales en Sector Turismo 2006. Lima.

PROMPERÚ. (2001). Perfil del profesional en turismo al 2021. Lima, PROMPERÚ.

PROMPERÚ. (2008). Perfil del vacacionista nacional, 2007. Lima, PROMPERÚ.

PROMPERÚ. (2009). Nivel de satisfacción del turista extranjero, 2008. Lima, PROMPERÚ. 